\title{
Dynamic Amyloid PET: Relationships to Flortaucipir Tau PET Measures
}

\section{Authors:}

Fabio Raman ${ }^{1,2,34}$; Yu-Hua Dean Fang PhD'; Sameera Grandhi, ${ }^{1,2}$; Charles F. Murchison, MS ${ }^{2,5}$; Richard E. Kennedy MD, PhD ${ }^{2,3}$; John C. Morris MD; ; Parinaz Massoumzadeh PhD; Tammie Benzinger MD, $\mathrm{PhD}^{7}$; Erik D. Roberson MD, $\mathrm{PhD}^{2,3,4}$, and Jonathan McConathy MD, $\mathrm{PhD}^{1,2 *}$

From 1 - The University of Alabama at Birmingham, Department of Radiology; 2 - The University of Alabama at Birmingham, Alzheimer's Disease Center; 3 - The University of Alabama at Birmingham, Department of Neurology; 4 - The University of Alabama at Birmingham, Center for Neurodegeneration and Experimental Therapeutics; 5 - The University of Alabama at Birmingham, Department of Medicine; 6 - Washington University in St. Louis School of Medicine, Department of Neurology; 7 - Washington University in St. Louis School of Medicine, Mallinckrodt Institute of Radiology,

\section{*Corresponding author:}

Jonathan McConathy M.D., Ph.D.

Department of Radiology

University of Alabama at Birmingham

$61919^{\text {th }}$ St S, Birmingham, AL 35249

E-mail: jmcconathy@uabmc.edu

Telephone: 205.934.1388

\section{${ }^{*}$ First author}

Fabio Raman (MD/PhD student)

E-mail: fabior@uab.edu

Telephone: 205.215.3669

Running Title: Hippocampal efAP predicts tau-PET

Manuscript word count: 5698 


\section{ABSTRACT}

Rationale: Measuring amyloid and predicting tau status using a single amyloid positron emission tomography (PET) study would be valuable for assessing brain AD pathophysiology. We hypothesized that early-frame amyloid PET (efAP) correlates with the presence of tau pathology because the initial regional brain concentrations of radioactivity are primarily determined by blood flow, which is expected to be decreased in the setting of tau pathology.

Methods: 120 participants (63 amyloid-positive/57 amyloid-negative) with dynamic ${ }^{18} \mathrm{~F}$ florbetapir-PET and static ${ }^{18} \mathrm{~F}$-flortaucipir-PET scans obtained within 6 months of each other were included. These subjects were predominantly cognitively intact in both the amyloid positive (63\%) and amyloid negative (93\%) groups. Parameters for efAP quantification were optimized for stratification of tau PET positivity, assessed by either a tauopathy score or Braak regions. The ability of efAP to stratify tau positivity was measured using receiver operating characteristics (ROC) analysis of area under the curve (AUC). Pearson's $r$ and Spearman's $\rho$ were used for parametric and non-parametric comparisons between efAP and tau PET, respectively. Standardized net benefit was used to evaluate improvement in utilizing efAP as an additional copredictor over hippocampal volumes in predicting tau PET positivity.

Results: Measuring efAP within the hippocampus and summing the first 3 minutes of brain activity post-injection showed the strongest discriminative ability to stratify for tau positivity (AUC 0.670.89 across tau PET Braak regions) in amyloid positive individuals. Hippocampal efAP correlated significantly with a global tau-PET tauopathy score in amyloid-positive participants $(r=-0.57, P<$ 0.0001). Compared to hippocampal volumes, hippocampal efAP showed stronger association with tau PET Braak stage ( $\rho=-0.58$ vs. -0.37 ) and superior stratification of tau PET tauopathy score (AUC: 0.86 vs. $0.66, P=0.002$ ).

Conclusions: Hippocampal efAP can provide additional information to conventional amyloidPET, including estimation of the likelihood of tau positivity in amyloid-positive individuals.

Keywords: ${ }^{18} \mathrm{~F}$-florbetapir-PET, ${ }^{18} \mathrm{~F}$-flortaucipir-PET, early phase PET, early-frame amyloid PET 


\section{INTRODUCTION}

Alzheimer's disease (AD) is the primary cause of age-related dementia, affecting approximately 50 million people worldwide. AD pathophysiological processes begin years before clinical symptoms. Established neuroimaging biomarkers include neurodegeneration with magnetic resonance imaging (MRI) and 2-deoxy-2[18F]fluoro-D-glucose ( $\left.{ }^{18} \mathrm{~F}-\mathrm{FDG}\right)$ positron emission tomography (PET), amyloid plaques and tau tangles with PET (1). These biomarkers can predict pathophysiological progression as well as cognitive performance $(1,2)$.

In standard amyloid-PET studies, amyloid status is measured 30-120 min after injection depending on the tracer $(3,4)$. Dynamic acquisition allows collection of a second potential biomarker during the initial tracer flow/delivery phase (5-7), but the clinical role of these early uptake measures is not well-defined. A number of groups have shown strong correlations between early flow measures from dynamic amyloid-PET and regional cerebral perfusion measured with ${ }^{15} \mathrm{O}$-water-PET $(6,8)$ and regional cerebral glucose metabolism measured with ${ }^{18} \mathrm{~F}$ FDG (9-11). Other studies have shown that higher tau burden measured with PET correlates with regional hypometabolism observed with ${ }^{18}$ F-FDG-PET $(12,13)$. This body of work led us to pursue the relationship between flow measures with dynamic amyloid-PET and the presence of tau pathology.

The objectives of this study were to determine the relationship between early frame amyloidPET (efAP) and tau PET in amyloid-positive individuals and to assess the potential of dynamic amyloid PET to predict tau-PET status. We chose a relatively simple method to calculate efAP to increase the clinical relevance and ease of implementation. We hypothesized that reduced efAP would correlate with pathologic tau measured by PET in amyloid-positive individuals evaluated with both dynamic amyloid and tau PET scans within 6 months of each other.

\section{MATERIALS AND METHODS}

\section{Study Population}


Participants were selected from a cohort at the Charles F. and Joanne Knight Alzheimer Disease Research Center (Knight ADRC) at Washington University in St. Louis (WUSTL). The recruitment, assessment and exclusion criteria methods have been published previously (14) and are available on at https://knightadrc.wustl.edu. All studies were approved by the Institutional Review Board at WUSTL and the University of Alabama at Birmingham, indicating compliance with all ethical regulations; informed consent was obtained from all participants before study enrollment.

From the cohort of 410 unique participants, 63 amyloid-positive and 57 amyloid-negative participants who met eligibility criteria were selected. Subjects had to have dynamic amyloidPET, tau-PET, and brain MRI completed within 6 months of each other and have at least 18months of neuropsychological evaluation after completion of imaging. Subjects were categorized as amyloid- and tau-positive or negative based on established cutoffs from the Knight ADRC $(15,16)$. Specifically, mean cortical standardized uptake value ratio (SUVR) of 1.19 for amyloid PET (16) and tauopathy SUVR of 1.22 for tau PET (15) were used to stratify amyloid and tau positivity and screen subjects based on prior analyses. Subject selection and demographics including cognitive measures are presented in Fig. 1 and Table 1, respectively.

\section{Image Sets Used for Analysis}

${ }^{18} \mathrm{~F}$-florbetapir-PET was performed using an intravenous bolus of $274-418 \mathrm{MBq}(7.4-$ $11.3 \mathrm{mCi}$ ) on a Siemens Biograph mMR scanner. Data were acquired at the time of injection through $70 \mathrm{~min}$, and reconstruction was performed with 26 frames $(4 \times 15 \mathrm{~s}, 4 \times 30 \mathrm{~s}, 3 \times 60 \mathrm{~s}, 3$ x 120 s, 2 x 240 s, 10 x 300 s). Data from 50-70 min post-injection was used for amyloid quantification (16). Amyloid SUVRs were calculated in brain ROIs using the entire cerebellum as the reference region.

$\mathrm{MRI}$ and ${ }^{18} \mathrm{~F}$-flortaucipir-PET acquisition and pre-processing were performed as previously reported (15). Tau-PET was performed on a Siemens Biograph 40 PET/CT scanner using an 
intravenous bolus of $266-400 \mathrm{MBq}(7.2-10.8 \mathrm{mCi})$ of ${ }^{18} \mathrm{~F}$-flortaucipir. Data from $80-100$ min postinjection were used for regional brain tau quantification (15). Tau SUVRs were calculated in brain ROls using cerebellar gray matter as the reference region.

\section{Calculation of Flow Phase Parameters from Dynamic Amyloid PET}

Regional dynamic amyloid and static tau PET data were measured using MR-based FreeSurfer segmentation (17) with the Biomarker Localization, Analysis, Visualization, Extraction and Registration (BLAzER) algorithm developed by our group (18). Calculation of efAP from dynamic amyloid PET was performed in Matlab vR2019b (MathWorks, Natick, MA). A set of timeaverage early-frame efAP intervals were generated in Matlab to test a range of different start points (5\% to $50 \%$ of peak cerebral cortex radioactivity) and end points of early-frame intervals (45 to 600 seconds from start of acquisition). A total of 79 target ( $T$ ) regions of interest (ROls) based on FreeSurfer segmentation were integrated over each early-frame interval and subsequently normalized to the integrated TAC of the appropriate reference $(R)$ region over the same period. Since discrete integrals were used based on the radioactivity $(A)$ and associated frame duration $(D)$ over the selected set of early-frames, the equation (Eq. 1) could be simplified as follows:

$$
(E q .1) \text { ef } A P_{\text {SUVR }}=\frac{\sum_{i=S t a r t}^{E n d}\left(A_{T i} D_{T i}\right)}{\sum_{i=S t a r t}^{E n d}\left(A_{R i} D_{R i}\right)}
$$

\section{Tau Assessment in Tauopathy Summary Measure, Braak Regions, and In Vivo Braak Staging}

A tauopathy summary measure was previously experimentally derived by using a sparse k-means clustering with resampling analysis to identify the regions most informative in dividing a cognitively normal population into high tau and low tau groups. The highest-weighted FreeSurfer regions of interest (ROIs) separating these groups were the entorhinal cortex, amygdala, lateral 
occipital cortex, and inferior temporal cortex, and an average SUVR in these four ROls was used as a summary metric for ${ }^{18} \mathrm{~F}$-flortaucipir uptake (15).

To replicate Braak neuropathological staging, tau-PET Braak regions were created from volume-weighted FreeSurfer subregions as defined by BLAzER (18). Braak regions refers to specific anatomic regions regardless of their tau status while Braak staging refers to the presence of pathologic tau in these regions based on ${ }^{18} \mathrm{~F}$-flortaucipir-PET. Similar to Scholl et al. 2016, tauPET Braak staging was performed by first categorizing the following volume-weighted, composite regions: transentorhinal (Braak stage I/II), limbic (III/IV), and isocortical (V/VI) (19). To maintain consistency across thresholding techniques, the same 1.22 cutoff (15) used to determine tau status was applied to the composite Braak regions as follows: subjects positive (SUVR > 1.22) across all three regions (I/II, III/IV, and V/VI) were assigned Braak stage $\mathrm{V} \& \mathrm{VI}$; subjects positive in I/II and III/IV, but negative in V/VI, were assigned Braak stage III \& IV; subjects positive in I/II, but negative elsewhere, were assigned Braak stage I \& II; and subjects negative across all three composite ROls were assigned Braak stage 0.

\section{Calculation of Normalized Hippocampal Volumes}

Hippocampal volumes were reported as a percentage of the cerebral cortex to normalize across different participants using the following equation (Eq. 2) (20):

$$
\text { (Eq. 2) } \frac{\text { Volume of hippocampus }}{\text { Volume of cerebral cortex }} * 100 \%
$$

\section{Statistical Analyses}

All statistical analyses were performed using SPSS Statistics v26.0 (IBM, Armonk, NY) and Matlab vR2019b (MathWorks, Natick, MA) to compare efAP values to tau-PET positivity. All parametric analyses between Braak regions used Pearson's $r$ correlation coefficient while nonparametric analyses across all Braak stages used Spearman's $\rho$ and between individual Braak 
stages used Wilcoxon ranked sum test. The significance level was a $P$ value of less than 0.05 with a Bonferroni correction for multiple comparisons.

Inter-subject, univariate Pearson correlations between efAP and tau were performed between efAP and tau PET Braak regions I - VI across all 120 subjects, separating subjects by amyloid status. Because only one subject was positive in tau PET Braak VI (SUVR > 1.22), this region was excluded for optimization purposes. Additionally, only the 63 amyloid-positive subjects were used for optimization purposes to align with our hypothesis. Area under the curve (AUC) was used to test the performance of the receiver operating characteristics (ROC) curve where efAP was used as the predictor variable and tau positivity (SUVR > 1.22) in tau PET Braak regions $\mathrm{I}-\mathrm{V}$ was used as the outcome variable. The early-frame interval and target region corresponding to the maximum AUC, mean \pm standard deviation (SD) was selected for the remainder of the study. Similarly, the following 6 reference regions for the calculation of efAP were compared for the ability to predict positive tau-PET: entire cerebellum, cerebral white matter, pre/post central gyrus, Braak VI tau region, Braak V/VI composite region, and basal ganglia.

Logistic regression models were used to examine the accuracy of efAP to distinguish between the tau negative and tau positive groups. Performance of efAP was assessed using ROC curves to compare the sensitivity and specificity and to determine the optimum cutoff point for efAP and best time interval. For parameter optimization for the dataset, AUC was calculated and compared to the value of 0.5 (random agreement) using the methods of Obuchowski et al (21). Sensitivity, specificity, and positive (PPV) and negative predictive value (NPV) were reported at the optimum cutoff based on maximum Youden's index (22). To evaluate performance between biomarkers in stratifying for tau positivity, ROC curves were compared using the methods of DeLong et al (23) with standard error as calculated by SPSS. Additionally, standardized net benefit (SNB) was used to measure the improvement in efAP as an additional predictor over hippocampal volumes with tau PET positivity set as the outcome variable $(24,25)$. 


\section{RESULTS}

\section{Selection of Optimum efAP Target and Reference Region}

Our first objective was to determine which brain region, reference region, and time frames would optimize the predictive performance of efAP. When evaluating efAP as the predictor variable for each of the 79 ROIs and tau positivity as the outcome variable, efAP in tau PET Braak II region (hippocampus), showed the strongest AUC across Braak regions I-V when using a cerebral white matter reference region $(0.79 \pm 0.082)$, peaking at 0.89 for tau PET using Braak region III (Fig. 2, Supplemental Figs. 1 and 2). Thus, hippocampal efAP was chosen as the target region and cerebral white matter was chosen as the reference region for efAP calculations for the remainder of the study.

\section{Selection of Optimum efAP Time Interval}

Next we determined which dynamic amyloid PET early time interval was most closely associated with tau PET. Using ROC analysis, the AUC values were compared when using hippocampal efAP as the predictor variable and tau PET positivity across tau PET Braak regions $\mathrm{I}-\mathrm{V}$ as the outcome variables (Supplemental Fig. 3). The endpoint of the early phase interval was determined to be ideal at 3 minutes post-injection where the AUC was highest $(0.79 \pm 0.0042)$. Selection of endpoint only showed 3.3\% decline from this peak AUC until 6 minutes post-injection and $8.5 \%$ decline until 10 min post-injection, emphasizing relative stability of efAP measurement to varying time intervals. Although altering the start point of the early phase interval showed only marginal variations in the AUC (<5\%), setting a higher threshold of peak cerebral cortex activity for selection of the first frame used in efAP measurement marginally decreased the AUC at all points (Supplemental Fig. 3). Thus, the early phase interval starting at $5 \%$ of cerebral cortex peak activity and ending at 3 minutes post-injection was deemed optimal.

\section{Hippocampal efAP Correlates with Tau PET Results in Amyloid-Positive Participants}


Next, we evaluated the relationships between hippocampal efAP and tau PET. A visual example of the inverse correlation between hippocampal efAP and tau PET for a normal subject (tau PET Braak Stage 0) and for late-stage disease (tau PET Braak Stage V \& VI) are shown in Fig. 3. Comparing hippocampal efAP to the tauopathy summary measure showed a significant Pearson correlation coefficient in amyloid-positive participants (Pearson $r=-0.57, P<0.0001$, As expected, this relationship was absent in amyloid-negative participants (Fig. 4). Significant regional correlations $(P<0.001)$ were also seen in amyloid-positive participants across tau PET Braak regions I $-\mathrm{V}(r=-0.50,-0.43,-0.58,-0.66$, and -0.48 , respectively, Supplemental Fig. 4). ROC analysis revealed that hippocampal efAP could be used to strongly predict tau positivity on tau PET in amyloid-positive participants at a global level using the tauopathy summary measure $(A \cup C=0.86$, efAP cutoff $=1.06$, sensitivity $=71 \%$, specificity $=93 \%$, PPV $=93 \%$, and NPV $=72 \%$, Fig. 5A). Regional analyses in individual Braak regions I-V also showed strong discrimination of tau PET positivity $(A U C=0.75,0.67,0.89,0.84$, and 0.80 , respectively, Supplemental Fig. 5).

\section{Hippocampal efAP Shows Stronger Correlation with Tau Status than hippocampal volumes}

Reduction in hippocampal volumes could reduce SUVs due to partial volume averaging. Therefore, we compared these two biomarkers in amyloid positive individuals to determine if hippocampal efAP conveyed additional information not provided by hippocampal volume measurement alone. Characterizing subjects by their tau PET Braak stage showed that both hippocampal efAP and hippocampal volumes significantly decreased with increasing tau PET Braak stage (Fig. 6). Hippocampal efAP showed a stronger inverse relationship with tau PET Braak stage $(\rho=-0.58, P<0.0001$, Fig. 6 A $)$ than hippocampal volumes $(\rho=-0.37, P=0.0034$, Fig. 6B). Wilcoxon ranked sum test between individual groups showed similar results with the differences between Braak stage I \& II and Braak stage III \& IV showing the greatest difference for both hippocampal efAP $(1.11 \pm 0.07$ vs. $1.00 \pm 0.07, P=0.0002$, Fig. $6 \mathrm{~A})$ and hippocampal 
volumes $(1.86 \pm 0.18$ vs. $1.60 \pm 0.20, P=0.0014$, Fig. $6 \mathrm{~B})$. When looking at efAP and hippocampal volume as individual predictors of tau PET, the discriminatory ability to predict tau positivity was superior for hippocampal efAP compared to hippocampal volumes across the tauopathy summary measure (AUC: 0.86 vs. $0.66, z=-3.08, P=0.002)$ with higher sensitivity $(71 \%$ vs. $46 \%)$, specificity (93\% vs. $89 \%)$, PPV (93\% vs $84 \%)$, and NPV ( $72 \%$ vs $57 \%) \quad$ (Fig. $5 \mathrm{~A})$. Although hippocampal efAP showed a trend towards higher predictive ability than hippocampal volume for Braak Stage III and greater, the results did not reach statistical significance (AUC: 0.87 vs. 0.76 , $z=1.54, P=0.12$, Fig. 5B).

Similarly, when grouping efAP and volumes as co-predictors to tau positivity, efAP showed a significant, additive benefit to simply using hippocampal volumes alone to predict tau positivity when utilizing tauopathy summary $\mathrm{ROI}(\triangle \mathrm{SNB}=0.3472, P=0.0070)$ but not for Braak Stage III and greater positivity $(\triangle \mathrm{SNB}=0.0998, P=0.38)$.

\section{DISCUSSION}

Hippocampal efAP, a biomarker related to flow during the early frames of dynamic amyloid PET, correlates with tau PET in amyloid-positive individuals. Hippocampal efAP shows promise for predicting tau pathology measured with ${ }^{18} \mathrm{~F}$-flortaucipir-PET and provides significant, additive utility in predicting tau pathology over hippocampal volumes alone. The use of efAP could add specificity to the assessment of $A D$ with amyloid-PET and allow a more comprehensive neuroimaging examination

Our cross-sectional retrospective analysis demonstrated a strong, significant association between hippocampal efAP and tau PET Braak stage (Fig. 6A). Our results also showed that hippocampal efAP had strong discriminatory performance in assessing tau positivity at the optimum cutoff, based on maximum Youden's index (22), whether using either a validated tauopathy measure (15) or Braak staging. For instance, hippocampal efAP had a high PPV (93\%) and moderate NPV (72\%) in amyloid-positive individuals compared to the tauopathy summary 
measure with flortaucipir-PET. The SUVR threshold for tau-positivity (1.22) used in this study was based on the previously conducted study at the Knight ADRC (15). This SUVR threshold may vary based on the population and PET study parameters but likely fall within a fairly narrow range based on studies by other groups who reported optimal cut-offs ranging from 1.23 to 1.27 (2628).

Among the 79 target regions evaluated for efAP, the hippocampus provided the strongest predictive power for tau positivity. The identification of the hippocampus as the most accurate target region for efAP was somewhat surprising as the precuneus and posterior cingulate gyrus show hypometabolism (29) and hypoperfusion (30) early in the course of AD. Previous research has mainly explored direct, one-to-one regional correlations between ${ }^{18} \mathrm{~F}-\mathrm{FDG}-\mathrm{PET}$ and tau PET $(12,13,31)$ and ${ }^{18}$ F-FDG-PET and perfusion $(9-11,32)$, and it is recognized that spatial differences exists between ${ }^{18}$ F-FDG and tau PET (31), such as in the hippocampus where hypometabolism does not correlate well with tau pathology (12). Alternative methods for estimating perfusion from dynamic amyloid PET exist, such as pharmacokinetic (PK) modeling (11,33). In particular, Joseph-Mathurin et al noted that PK-derived R1 values from ${ }^{11} \mathrm{C}-\mathrm{PiB}$ spatially correlated with ${ }^{18} \mathrm{~F}$ FDG-PET but early-frame intervals did not (33).

We found the optimal early-frame time window started at $5 \%$ of peak cerebral cortex activity and ended at 180 seconds post-injection. Importantly, efAP was found to be relatively stable up to 6 and 10 minutes with small reductions in AUC reduction. Our results were similar to another ${ }^{18} \mathrm{~F}$-florbetapir study that evaluated the performance of early-frame dynamic amyloid PET imaging compared to ${ }^{18} \mathrm{~F}-\mathrm{FDG}-\mathrm{PET}$, in which the $1-6$-minute time window provided the best surrogate for perfusion based on ${ }^{18} \mathrm{~F}-\mathrm{FDG}-\mathrm{PET}$ (32). Several other studies that utilized ${ }^{11} \mathrm{C}-\mathrm{PiB}$ instead of ${ }^{18} \mathrm{~F}$-florbetapir showed similar results $(6,10,11,34)$. One of these studies in particular corroborated our results by finding that a shorter interval, specifically $20-130$ seconds postinjection, best discriminated between $A D$ patients and controls despite a longer interval correlating better with ${ }^{18} \mathrm{~F}-\mathrm{FDG}-\mathrm{PET}$ (10). Our efAP method focused on quantitative analysis rather than 
visual interpretation, and it is possible a longer time interval would be more suitable for visual analysis by reducing image noise.

Reduction in hippocampal volume, as measured by MR, has been robustly validated and remains one of the core biomarkers in AD due to strong evidence supporting its diagnostic and prognostic value $(2,35)$. In our present study, decreases in both hippocampal efAP and hippocampal volumes were shown to be inversely correlated with tau PET Braak staging Hippocampal eFAP was a better predictor of tau positivity based on the tauopathy summary measure than hippocampal volumes with additive benefit over hippocampal volume measurements alone. A trend towards better performance with hippocampal efAP compared to hippocampal volume $(P=0.12)$ was observed for Braak stage III and higher but did not reach statistical significance. The relatively small number of participants with elevated tau in Braak regions III and higher $(n=21)$ prevents a definitive conclusion regarding the additional value of hippocampal efAP versus hippocampal volumes for individual Braak stages.

Our work has limitations that are important to address in future work. Our study population included a large proportion of amyloid-positive subjects who were cognitively normal or had mild cognitive impairment. Our efAP results including the optimal brain region for efAP measurement may be different in individuals with more advanced AD. We chose to focus on amyloid-positive subjects for the hippocampal efAP analysis as ${ }^{18} \mathrm{~F}$-flortaucipir is best suited for imaging the pathologic form of tau that is deposited in AD. Our evaluation of the amyloid-negative subjects was more limited as this is a potentially heterogeneous group that is not expected to have many individuals positive for tau based on ${ }^{18} \mathrm{~F}$-flortaucipir-PET. The PET tracer injection technique was not optimized for the early-frame measurement of efAP. Although exclusion criteria did help by removing subjects if the bolus perfusion phase was inadequate based on their time to peak (>150 seconds), the time to peak for included subjects still varied widely from 45-150 seconds. Our findings suggest that efAP was not highly sensitive to differences in rate of bolus delivery, but future studies would benefit from a more standardized injection technique. 


\section{CONCLUSION}

We have shown that efAP acquired concurrently with standard amyloid PET study is a strong predictor of tau pathology in amyloid-positive individuals. Successful development of this approach has the potential to provide information on both amyloid and tau pathology in a single PET session which may reduce imaging costs and burden on patients and their families.

\section{DISCLOSURE}

\section{Funding Sources}

This work was supported by National Institutes of Health grants P20AG068024, RF1AG059009 and T32GM008361 Medical Science Training Program, the Alzheimer's Drug Discovery Foundation, and the Department of Radiology at the University of Alabama at Birmingham. Tammie Benzinger is funded by NIH grants P50AG005681; P01AG003991; P01AG026276; UF01AG032438, R01AG05326, RF1AG053550, R01AG054567, R01AG052550. John C. Morris is funded by NIH grants P50AG005681; P01AG003991; P01AG026276 and UF01AG032438. The recruitment of participants and their clinical characterization and neuroimaging were supported by NIH grants P50AG05681, P01AG03991, and P01AG026276.

\section{Conflicts of Interests}

Avid Radiopharmaceuticals, a wholly owned subsidiary of Eli Lilly $\left({ }^{18} \mathrm{~F}\right.$-florbetapir and ${ }^{18} \mathrm{~F}$ flortaucipir imaging). For ${ }^{18} \mathrm{~F}$-florbetapir, Avid provided the doses and partial support for scanning through an investigator-initiated research grant awarded to Washington University (J.C. Morris, T.L.S. Benzinger). Avid Radiopharmaceuticals (a wholly owned subsidiary of Eli Lilly) provided

technology transfer and provided precursor for ${ }^{18} \mathrm{~F}$-florbetapir. J. McConathy has declared a relationship with Eli Lilly and Avid to whom he provides consulting and from which he receives research support. No other potential conflicts of interest relevant to this article exist. 


\section{ACKNOWLEDGMENTS}

Data used in the preparation of this article were obtained from the WUSTL Knight ADRC and are available upon request (https://knightadrc.wustl.edu/). ${ }^{18} \mathrm{~F}$-flortaucipir was produced under a material transfer agreement between Washington University and Avid Radiopharmaceuticals. 


\section{KEY POINTS}

- QUESTION: Do the first few frames after tracer injection on dynamic amyloid PET predict tau pathology on tau-PET in amyloid positive participants?

- PERTINENT FINDINGS: This retrospective study showed that decreased activity in the hippocampus measured with early frame amyloid PET (efAP) within predicts tau pathology on tau-PET in amyloid-positive individuals.

- IMPLICATIONS FOR PATIENT CARE: Our findings show that efAP may facilitate prediction of tau status from an amyloid-PET study and provide a more comprehensive neuroimaging assessment of cognitive impairment with increased specificity for $A D$. 


\section{REFERENCES}

1. Jack CR, Jr., Bennett DA, Blennow K, et al. NIA-AA Research Framework: Toward a biological definition of Alzheimer's disease. Alzheimers Dement. 2018;14:535-562.

2. Jack CR, Jr., Wiste HJ, Weigand SD, et al. Age-specific and sex-specific prevalence of cerebral beta-amyloidosis, tauopathy, and neurodegeneration in cognitively unimpaired individuals aged 50-95 years: a cross-sectional study. Lancet Neurol. 2017;16:435-444.

3. Richards D, Sabbagh MN. Florbetaben for PET imaging of beta-amyloid plaques in the brain. Neurol Ther. 2014;3:79-88.

4. Wong DF, Rosenberg PB, Zhou Y, et al. In vivo imaging of amyloid deposition in Alzheimer disease using the radioligand ${ }^{18} \mathrm{~F}-\mathrm{AV}-45$ (florbetapir F 18). J Nucl Med. 2010;51:913920.

5. Ossenkoppele R, Prins ND, van Berckel BN. Amyloid imaging in clinical trials. Alzheimers Res Ther. 2013;5:36.

6. Blomquist $\mathrm{G}$, Engler $\mathrm{H}$, Nordberg A, et al. Unidirectional influx and net accumulation of PIB. Open Neuroimag J. 2008;2:114-125.

7. Forsberg A, Engler H, Blomquist G, Langstrom B, Nordberg A. The use of PIB-PET as a dual pathological and functional biomarker in AD. Biochim Biophys Acta. 2012;1822:380-385. 
8. Chen YJ, Rosario BL, Mowrey W, et al. Relative ${ }^{11} \mathrm{C}-\mathrm{PiB}$ delivery as a proxy of relative CBF: quantitative evaluation using single-session ${ }^{15} \mathrm{O}$-water and ${ }^{11} \mathrm{C}-\mathrm{PiB} \mathrm{PET}$. J Nucl Med. 2015;56:1199-1205.

9. Rodriguez-Vieitez E, Leuzy A, Chiotis K, Saint-Aubert L, Wall A, Nordberg A. Comparability of $\left[{ }^{18} \mathrm{~F}\right]$ THK5317 and $\left[{ }^{11} \mathrm{C}\right] \mathrm{PIB}$ blood flow proxy images with $\left[{ }^{18} \mathrm{~F}\right] \mathrm{FDG}$ positron emission tomography in Alzheimer's disease. J Cereb Blood Flow Metab. 2017;37:740-749.

10. Peretti DE, Vallez Garcia D, Reesink FE, et al. Relative cerebral flow from dynamic PIB scans as an alternative for FDG scans in Alzheimer's disease PET studies. PLoS One. 2019;14:e0211000.

11. Rodriguez-Vieitez E, Carter SF, Chiotis K, et al. Comparison of Early-Phase ${ }^{11} \mathrm{C}$ Deuterium-I-Deprenyl and ${ }^{11} \mathrm{C}-P$ ittsburgh Compound B PET for Assessing Brain Perfusion in Alzheimer Disease. J Nucl Med. 2016;57:1071-1077.

12. Bischof $\mathrm{GN}$, Jessen $\mathrm{F}$, Fliessbach $\mathrm{K}$, et al. Impact of tau and amyloid burden on glucose metabolism in Alzheimer's disease. Ann Clin Transl Neurol. 2016;3:934-939.

13. Whitwell JL, Graff-Radford J, Tosakulwong N, et al. Imaging correlations of tau, amyloid, metabolism, and atrophy in typical and atypical Alzheimer's disease. Alzheimers Dement. 2018;14:1005-1014. 
14. Berg L, McKeel DW, Jr., Miller JP, et al. Clinicopathologic studies in cognitively healthy aging and Alzheimer's disease: relation of histologic markers to dementia severity, age, sex, and apolipoprotein E genotype. Arch Neurol. 1998;55:326-335.

15. Mishra S, Gordon BA, Su Y, et al. AV-1451 PET imaging of tau pathology in preclinical Alzheimer disease: Defining a summary measure. Neuroimage. 2017;161:171-178.

16. Su Y, Flores S, Wang G, et al. Comparison of Pittsburgh compound B and florbetapir in cross-sectional and longitudinal studies. Alzheimers Dement (Amst). 2019;11:180-190.

17. Fischl B, Salat DH, Busa E, et al. Whole brain segmentation: automated labeling of neuroanatomical structures in the human brain. Neuron. 2002;33:341-355.

18. Raman F, Grandhi S, Murchison CF, et al. Biomarker Localization, Analysis, Visualization, Extraction, and Registration (BLAzER) Methodology for Research and Clinical Brain PET Applications. J Alzheimers Dis. 2019;70:1241-1257.

19. Scholl M, Lockhart SN, Schonhaut DR, et al. PET imaging of tau deposition in the aging human brain. Neuron. 2016;89:971-982.

20. Risacher SL, Anderson WH, Charil A, et al. Alzheimer disease brain atrophy subtypes are associated with cognition and rate of decline. Neurology. 2017;89:2176-2186. 
21. Obuchowski NA, Lieber ML, Wians FH, Jr. ROC curves in clinical chemistry: uses, misuses, and possible solutions. Clin Chem. 2004;50:1118-1125.

22. Habibzadeh $F$, Habibzadeh $\mathrm{P}$, Yadollahie M. On determining the most appropriate test cut-off value: the case of tests with continuous results. Biochem Med (Zagreb). 2016;26:297307.

23. DeLong ER, DeLong DM, Clarke-Pearson DL. Comparing the areas under two or more correlated receiver operating characteristic curves: a nonparametric approach. Biometrics. 1988;44:837-845.

24. Vickers AJ, Elkin EB. Decision curve analysis: a novel method for evaluating prediction models. Med Decis Making. 2006;26:565-574.

25. Pepe MS, Fan J, Feng Z, Gerds T, Hilden J. The net reclassification index (NRI): a misleading measure of prediction improvement even with independent test data sets. Stat Biosci. 2015;7:282-295.

26. McSweeney M, Pichet Binette A, Meyer PF, et al. Intermediate flortaucipir uptake is associated with Abeta-PET and CSF tau in asymptomatic adults. Neurology. 2020;94:e1190e1200. 
27. Ossenkoppele R, Rabinovici GD, Smith $\mathrm{R}$, et al. Discriminative accuracy of $\left[{ }^{18} \mathrm{~F}\right]$ flortaucipir positron emission tomography for alzheimer disease vs other neurodegenerative disorders. JAMA. 2018;320:1151-1162.

28. Dodich A, Mendes A, Assal F, et al. The A/T/N model applied through imaging biomarkers in a memory clinic. Eur J Nucl Med Mol Imaging. 2020;47:247-255.

29. Kato T, Inui Y, Nakamura A, Ito K. Brain fluorodeoxyglucose (FDG) PET in dementia. Ageing Res Rev. 2016;30:73-84.

30. Ishii K, Sasaki M, Yamaji S, Sakamoto S, Kitagaki H, Mori E. Demonstration of decreased posterior cingulate perfusion in mild Alzheimer's disease by means of $\mathrm{H}_{2}{ }^{15} \mathrm{O}$ positron emission tomography. Eur J Nucl Med. 1997;24:670-673.

31. Ossenkoppele R, Schonhaut DR, Scholl M, et al. Tau PET patterns mirror clinical and neuroanatomical variability in Alzheimer's disease. Brain. 2016;139:1551-1567.

32. Hsiao IT, Huang CC, Hsieh CJ, et al. Correlation of early-phase ${ }^{18} \mathrm{~F}$-florbetapir (AV45/Amyvid) PET images to FDG images: preliminary studies. Eur J Nucl Med Mol Imaging. 2012;39:613-620.

33. Joseph-Mathurin N, Su Y, Blazey TM, et al. Utility of perfusion PET measures to assess neuronal injury in Alzheimer's disease. Alzheimers Dement (Amst). 2018;10:669-677. 
34. Gietl AF, Warnock G, Riese F, et al. Regional cerebral blood flow estimated by early PiB uptake is reduced in mild cognitive impairment and associated with age in an amyloiddependent manner. Neurobiol Aging. 2015;36:1619-1628.

35. Pini L, Pievani M, Bocchetta M, et al. Brain atrophy in Alzheimer's disease and aging. Ageing Res Rev. 2016;30:25-48.

36. Morris JC. The Clinical Dementia Rating (CDR): current version and scoring rules. Neurology. 1993;43:2412-2414.

37. Folstein MF, Folstein SE, McHugh PR. "Mini-mental state". A practical method for grading the cognitive state of patients for the clinician. J Psychiatr Res. 1975;12:189-198. 


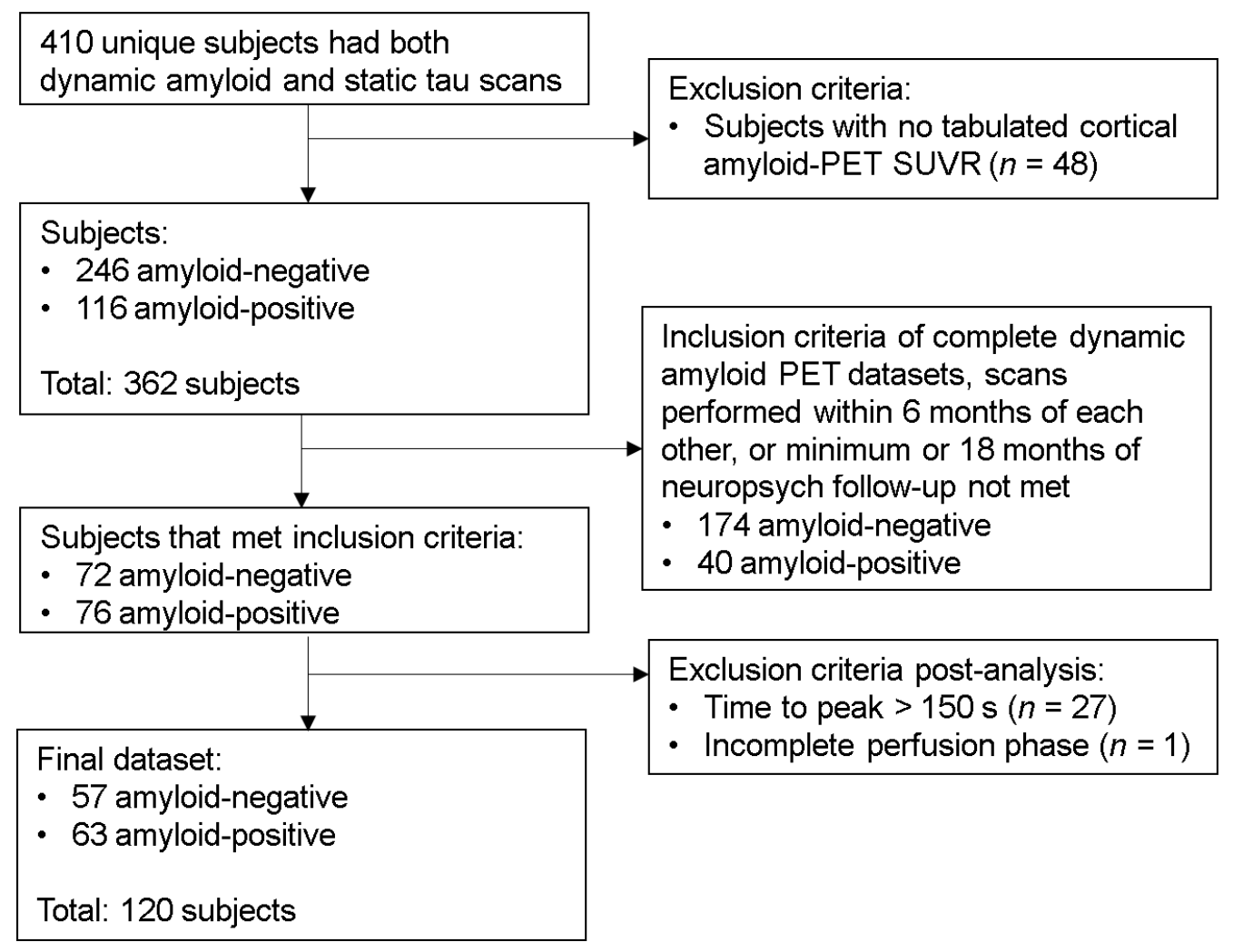

FIGURE 1. Subject selection diagram. Schematic flow diagram for selection of 63 amyloidpositive and 57 amyloid-negative subjects for analysis in this study from a retrospective cohort of 410 subjects. 
A

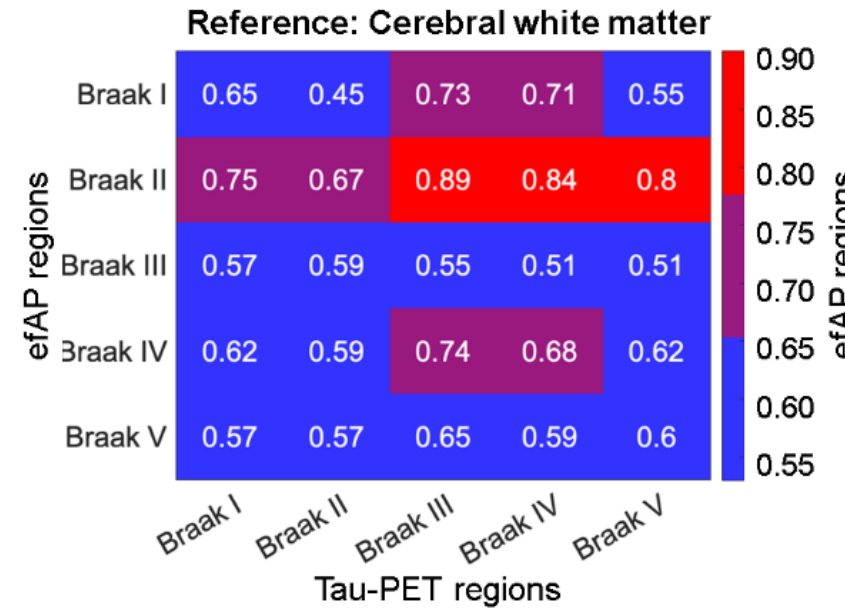

B

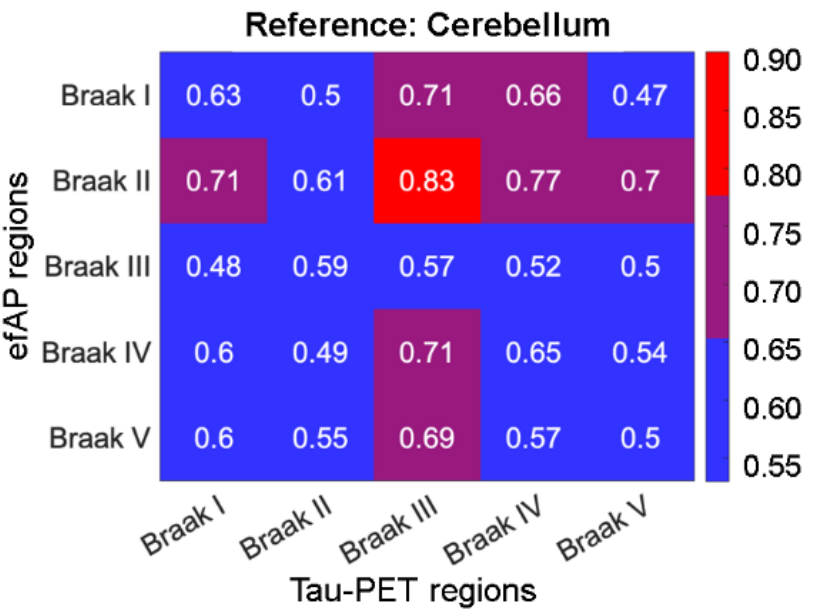

FIGURE 2. efAP Braak II region (hippocampus) and cerebral white matter chosen as target and reference regions, respectively. Area under the curve (AUC) was used to test the performance of the receiver operating characteristics (ROC) curve. AUC between the first 5 tau PET Braak regions shown for efAP being used as the predictor variable and tau PET as the outcome variable. Different reference regions for the calculation of efAP were compared with (A) cerebral white matter and (B) cerebellum showing the strongest AUC across efAP in Braak II region, also known as the hippocampus. The color scale shows ranges of AUC values in the tables with blue being lowest, purple intermediate, and red highest. 


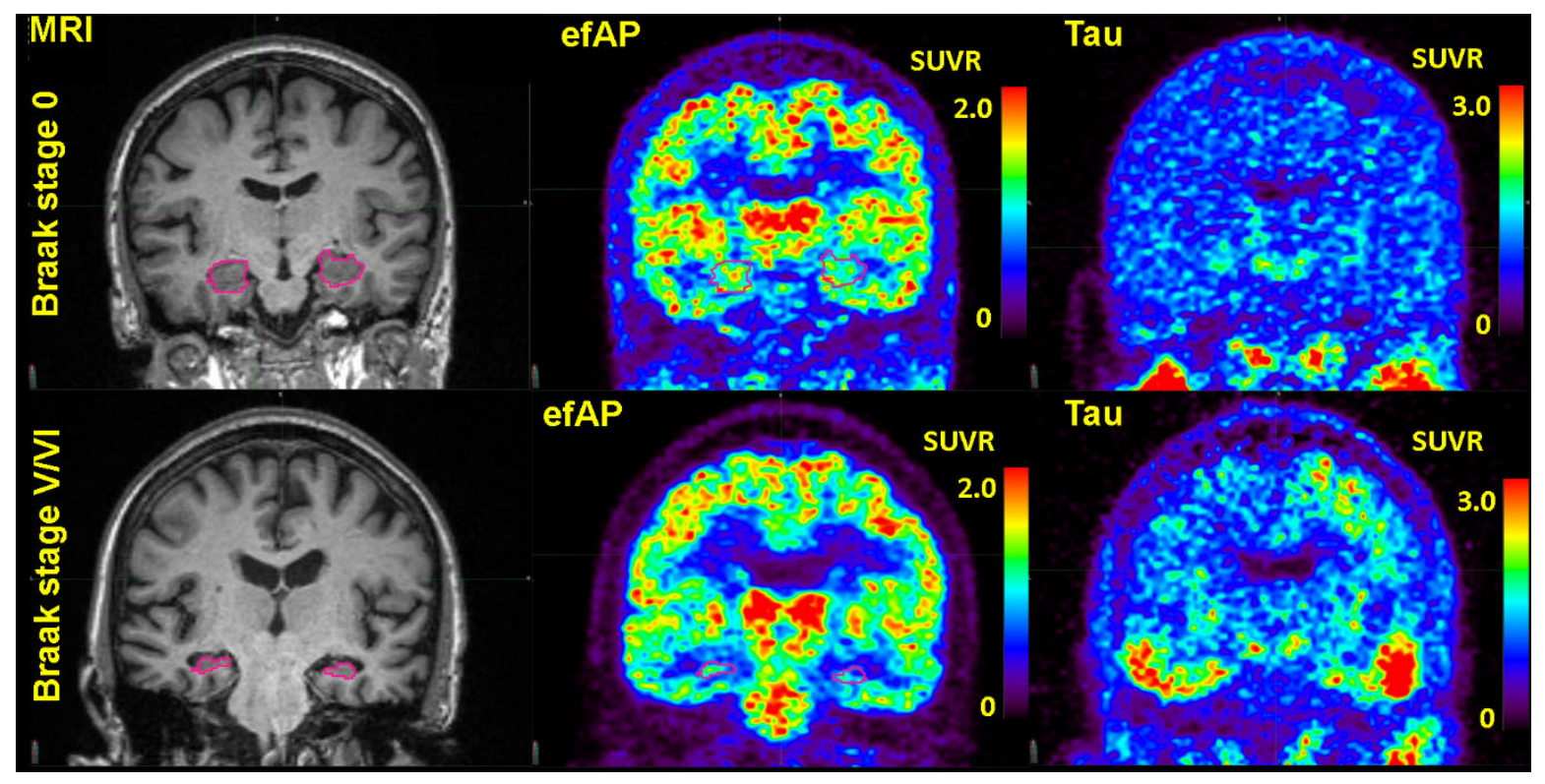

FIGURE 3. Example of inverse relationship between hippocampal efAP and tau PET Braak stage. Comparisons of representative subjects from tau PET Braak stage 0 (top) and Braak stage $\mathrm{V} \& \mathrm{VI}$ (bottom) show appearance of efAP at the two ends disease spectrum. The first column highlights the hippocampus in pink on volumetric MRI, the second column shows efAP values from the dynamic florbetapir-PET, and the third column shows tau PET. Intensity-scales for the PET images are shown as SUVRs. 


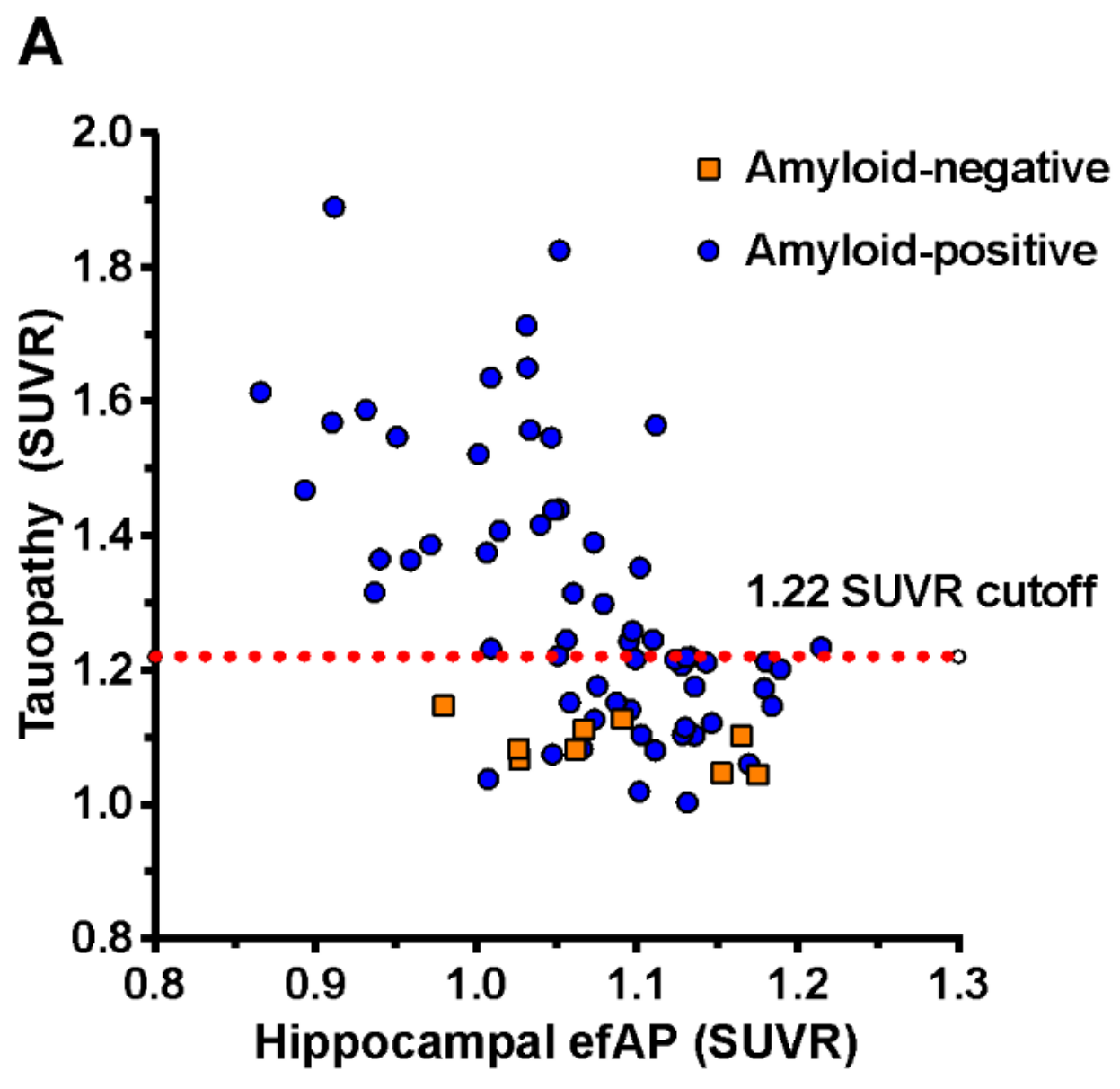

FIGURE 4. efAP parallels and predicts tau PET pathology. Comparison of hippocampal efAP and tau PET tauopathy summary measure are shown for amyloid-positive $(r=-0.57, P<0.0001)$ and negative $(r=0.05, P=0.69)$ participants. Tau PET SUVR cutoff used to stratify subjects for tau positivity is shown with dotted red line. 

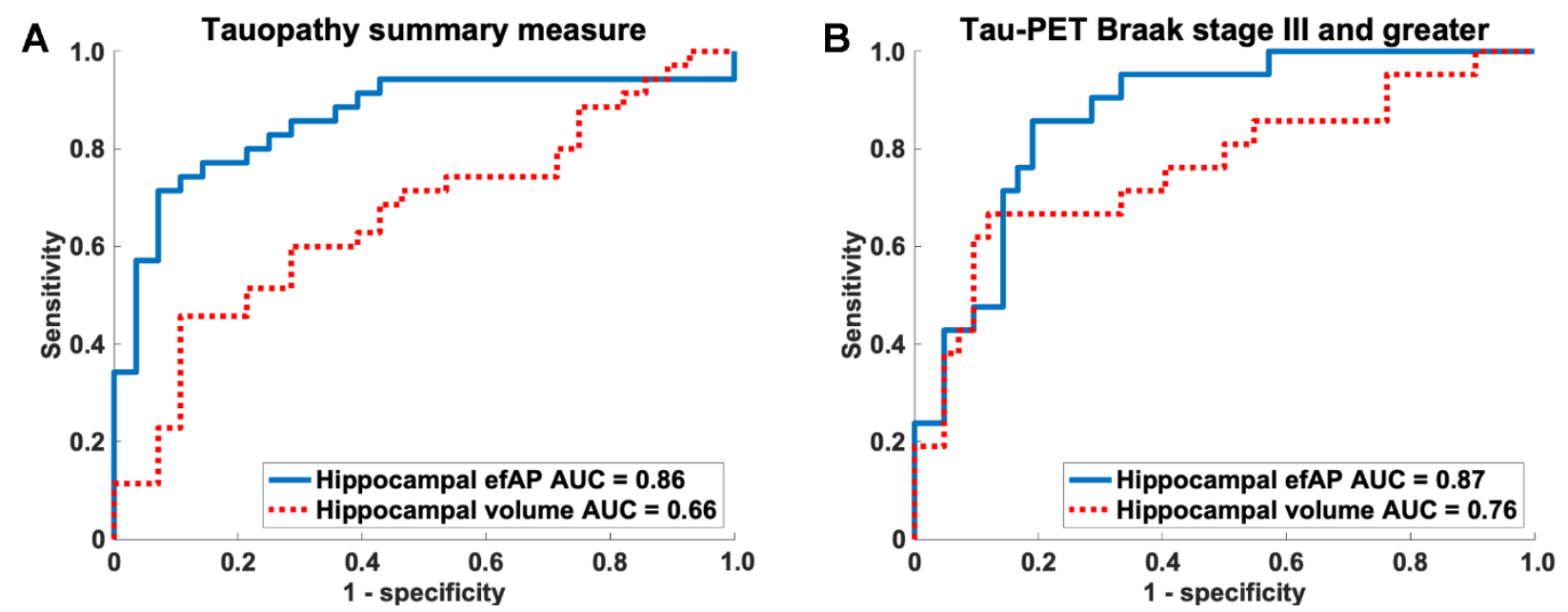

FIGURE 5. Hippocampal efAP has stronger discriminatory ability than hippocampal volume for predicting tau PET positivity in amyloid-positive subjects. Receiver operating characteristics curves shown for efAP (solid blue) and normalized hippocampal volumes (dashed red) as predictor variables and tau PET positivity in tauopathy summary measure and Braak stage III and greater as outcome variables. Tau-positivity was defined as SUVR $>1.22$. A) Area under the curve (AUC) measured for efAP cutoff of 1.06 was 0.86 with sensitivity and specificity of $71 \%$ and $93 \%$, and PPV and NPV of $93 \%$ and $72 \%$ respectively, and AUC measured for volumes was 0.66 with sensitivity and specificity of $46 \%$ and $89 \%$, and PPV and NPV of $84 \%$ and $57 \%$ respectively. AUC difference was statistically significant $(z=3.08, P=0.002)$. B) AUC measured for efAP cutoff of 1.05 was 0.87 with sensitivity and specificity of $86 \%$ and $81 \%$, with PPV and NPV of $69 \%$ and $92 \%$ respectively, and AUC measured for volumes was 0.76 with sensitivity and specificity of $67 \%$ and $88 \%$ and PPV and NPV of $74 \%$ and $84 \%$, respectively. This AUC difference did not reach statistical significance $(z=1.54, P=0.12)$. 


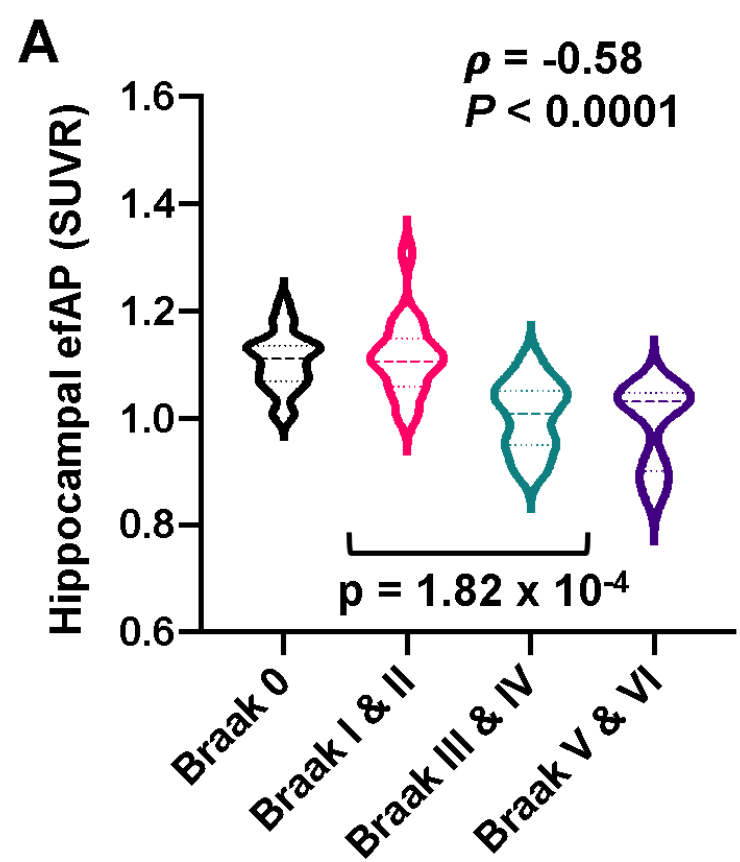

Tau-PET Braak stage

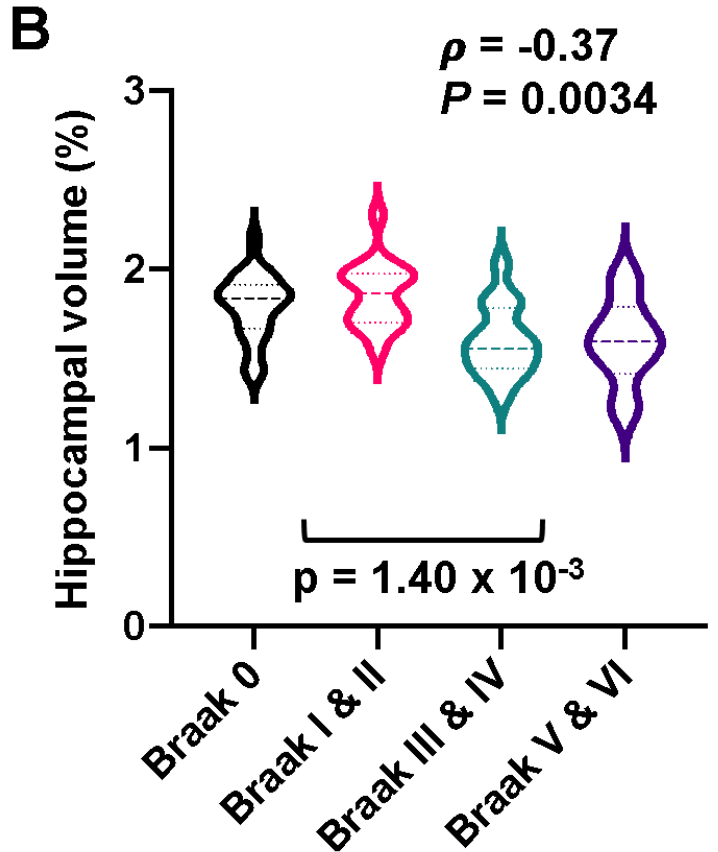

Tau-PET Braak stage

FIGURE 6. Tau PET Braak stage shows a stronger association with efAP than volumes within hippocampus. Amyloid-positive participants stratified by tau PET Braak stage and violinplots displayed for (A) hippocampal efAP and (B) normalized hippocampal volumes. Spearman rank $\rho$ used to test for association across all groups and Wilcoxon ranked sum test used to test for association between individual groups with $P<0.05$ denoted as significant. 


\begin{tabular}{|l|l|l|}
\hline & Amyloid-positive & Amyloid-negative \\
\hline $\mathbf{N}$ & 63 & 57 \\
\hline Tauopathy (+/-)* & $36 / 27$ & $7 / 50$ \\
\hline Age (years) & $74.36 \pm 7.95$ & $69.67 \pm 7.71$ \\
\hline Sex (M/F) & $25 / 33$ & $24 / 31$ \\
\hline Education & $15.97 \pm 2.55$ & $15.85 \pm 2.19$ \\
\hline APOE4 (0/1/2 \&4 alleles) & $24 / 27 / 7$ & $44 / 10 / 1$ \\
\hline MMSE (range) & $28.10 \pm 2.83[18,30]$ & $29.38 \pm 1.01[25,30]$ \\
\hline CDR (0/0.5/1) & $40 / 14 / 4$ & $53 / 2 / 0$ \\
\hline $\begin{array}{l}\text { Days in between amyloid } \\
\text { and tau PET scans }\end{array}$ & $63.17 \pm 67.86$ & $63.93 \pm 60.84$ \\
\hline
\end{tabular}

TABLE 1. Cohort demographics. Out of the subjects used in the study, 5 amyloid-positive and 2 amyloid-negative were missing demographic data and not included in the table. Number of total subjects $(N)$, broken down by sex with number of males $(M)$ and females $(F)$ shown. Apolipoprotein E- $\varepsilon 4$ (ApoE4) carriers represented as number of $\varepsilon 4$ alleles where 2 stands for homozygotes $(\varepsilon 4 / \varepsilon 4)$ and 1 for heterozygotes $\left(\varepsilon 4 / \varepsilon^{*}\right)$. Clinical Dementia Rating (CDR) score is based on a 5 point scale where subjects used were either 0 (not demented), 0.5 (uncertain or very mild dementia), or 1 (mild dementia) (36). All other results shown as mean \pm standard deviation. Mini-Mental State Examination (MMSE) scores range from 30 ("best') to 0 ("worst") with range of values shown in brackets for each group (37). Education is displayed at total years of formal education. 
Dynamic amyloid-PET time-activity curve
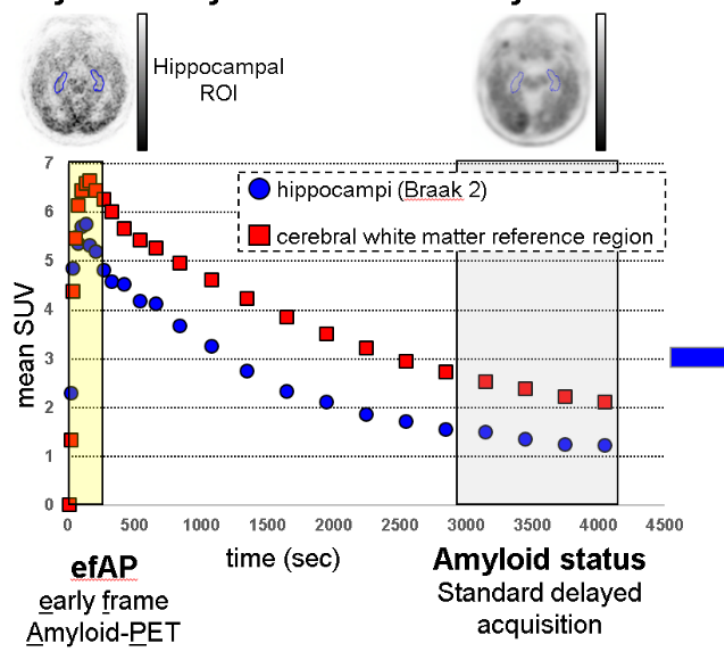

Reduced efAP correlates with pathological tau measured with PET in amyloid-positive individuals

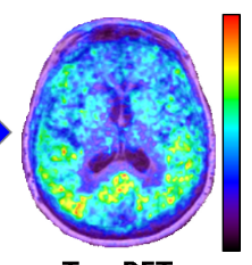

Tau-PET

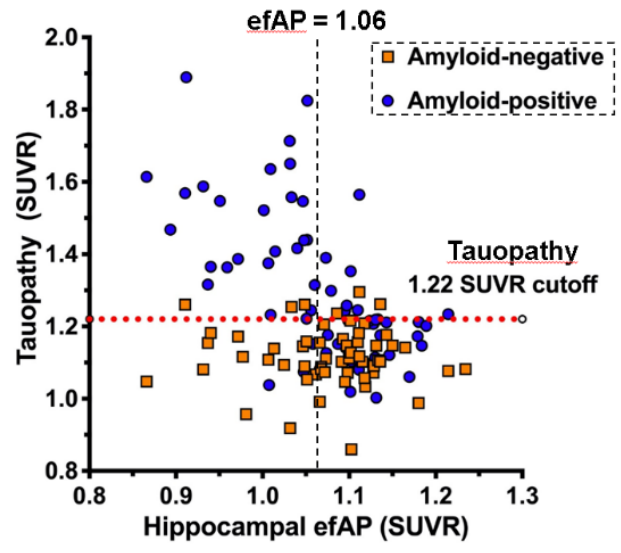

\section{Graphical Abstract}




\section{SUPPLEMENTAL DATA}

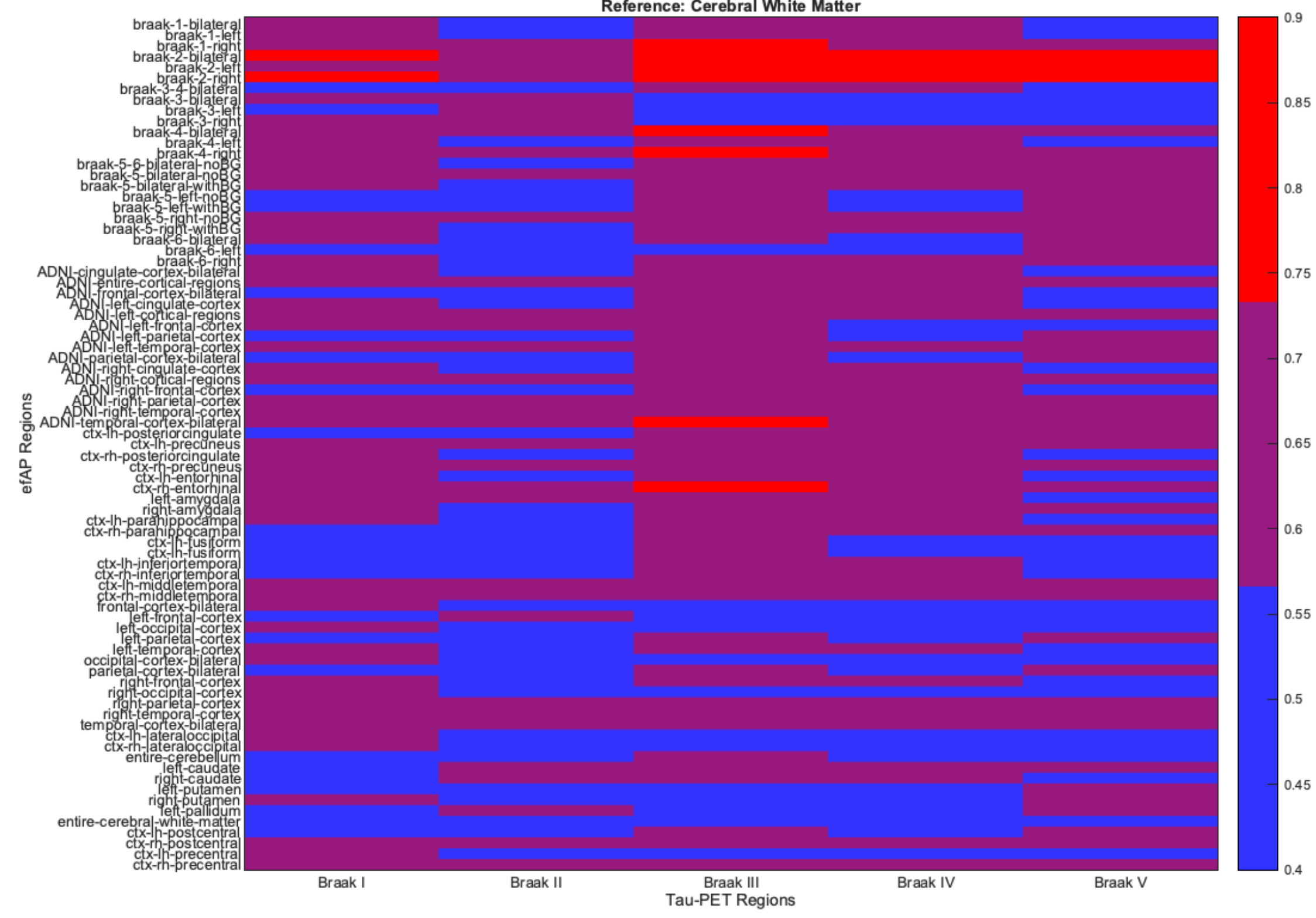


SUPPLEMENTAL FIGURE 1. efAP Braak II region (hippocampus) chosen as optimum target region. Area under the curve (AUC) was used to test the performance of the receiver operating characteristics (ROC) curve. AUC between the 79 efAP target regions being used as the predictor variable and tau PET Braak regions as the outcome variable. The color scale shows ranges of AUC values in the tables with blue being lowest, purple intermediate, and red highest. 
A)

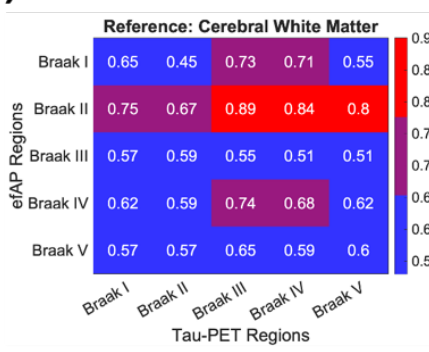

D)

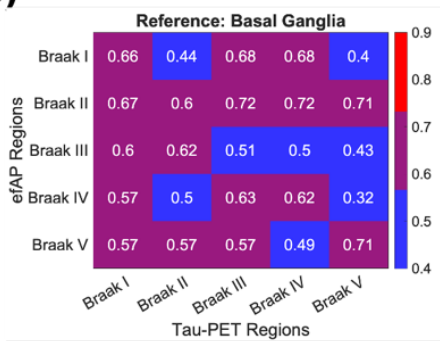

B)

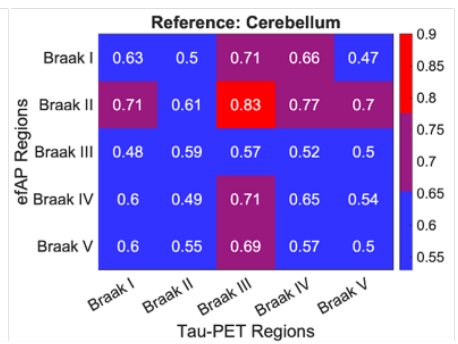

E)

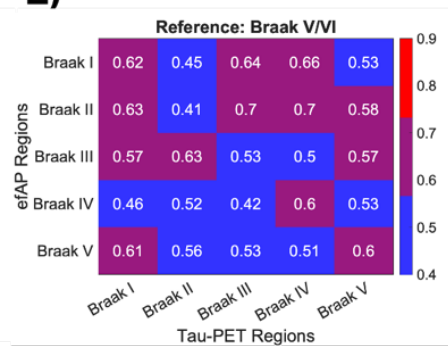

C)

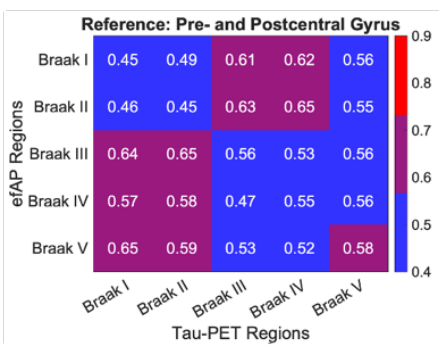

F)

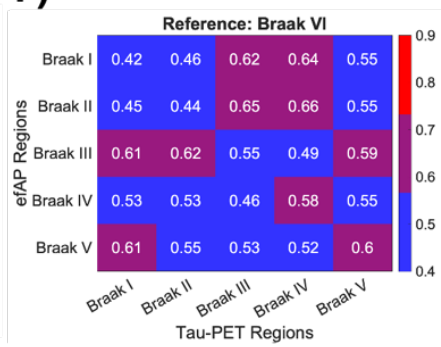

SUPPLEMENTAL FIGURE 2. efAP cerebral white matter chosen as optimum reference region. Area under the curve (AUC) was used to test the performance of the receiver operating characteristics (ROC) curve. AUC between the first 5 tau PET Braak regions shown for efAP being used as the predictor variable and tau PET as the outcome variable. Different reference regions for the calculation of efAP were compared: (A) cerebral white matter, (B) cerebellum, (C) pre- and postcentral gyrus, (D) basal ganglia, (E) tau PET Braak V and VI, and (F) Braak VI. The cerebral white matter showed the strongest AUC across efAP in Braak II target region, also known as the hippocampus The color scale shows ranges of AUC values in the tables with blue being lowest, purple intermediate, and red highest. 


\section{Optimization of Early-Phase Interval}

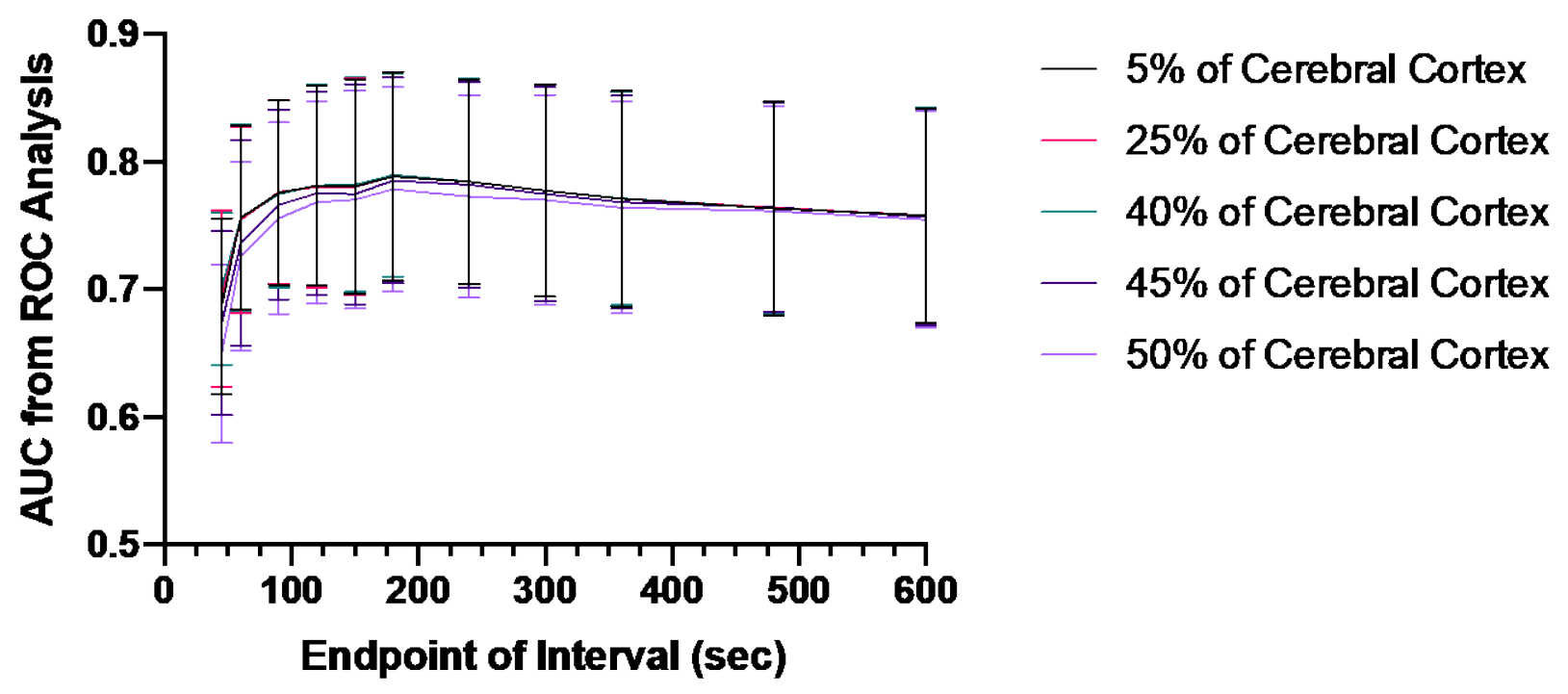

SUPPLEMENTAL FIGURE 3. First 180 seconds chosen as ideal efAP interval. Representative data from early-phase optimization of efAP shown from 5 different start points based on peak cerebral cortex activity across 11 duration intervals. Area under the curve (AUC) were measured from receiver operating characteristics (ROC) curves when using efAP as predictor and tau PET Braak regions $\mathrm{I}-\mathrm{V}$ as the outcome variable (mean \pm standard deviation across 5 Braak regions). 
A)

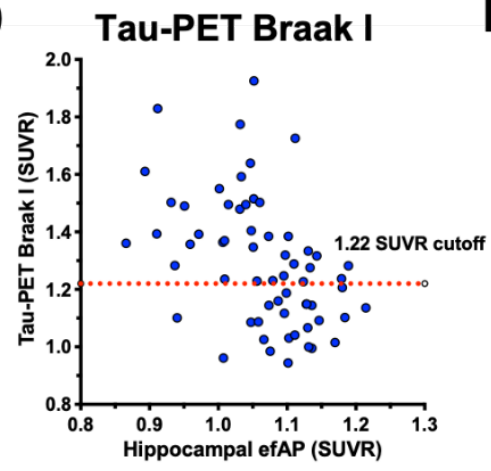

B)

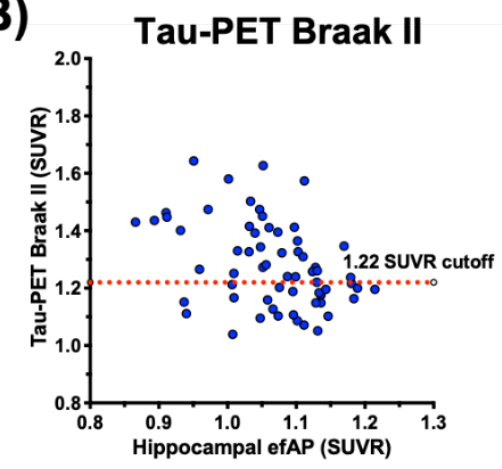

C) Tau-PET Braak III

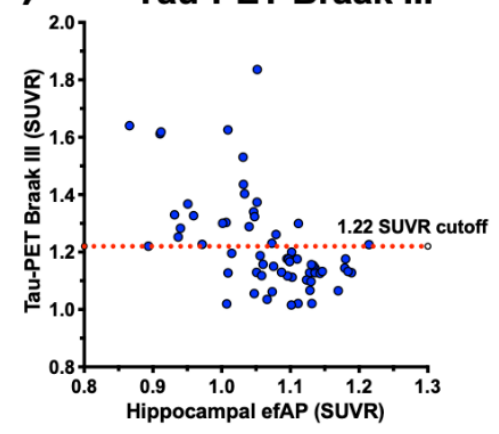

D)

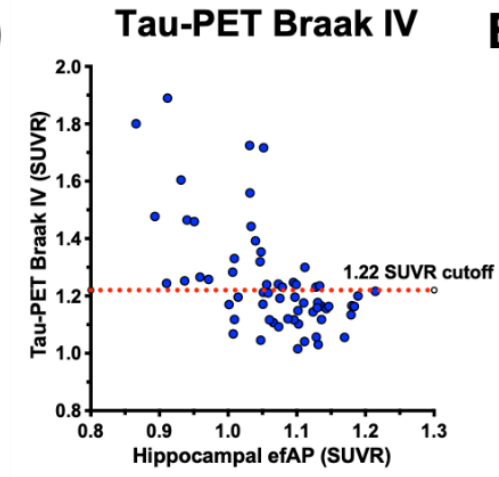

E)

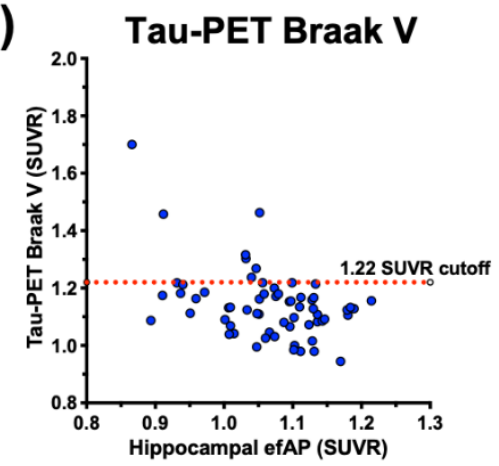

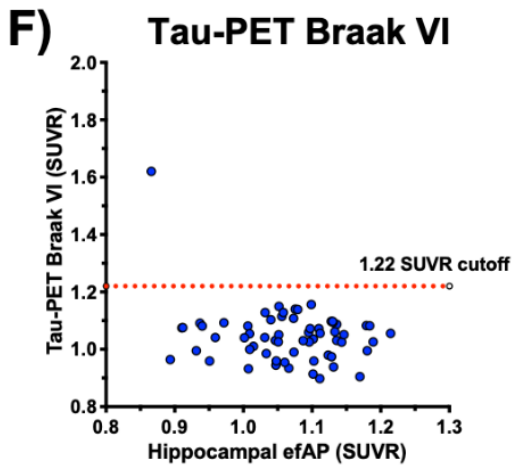

SUPPLEMENTAL FIGURE 4. Hippocampal efAP significantly correlated with tau PET Braak regions I through V. Comparison of efAP and flortaucipir-PET across the regions representing the pathological tau PET Braak stages for amyloid-positive participants. (A) through (F) represent the regions for Braak stages I through VI, respectively. Univariate Pearson correlation showed significant correlations with Braak regions I through $\vee(r=-0.50,-0.43,-0.58,-0.66$, and -0.48 , $P<0.001)$. Note that only 1 individual in the study was tau-PET positive in Braak VI, preventing meaningful correlation with efAP. 

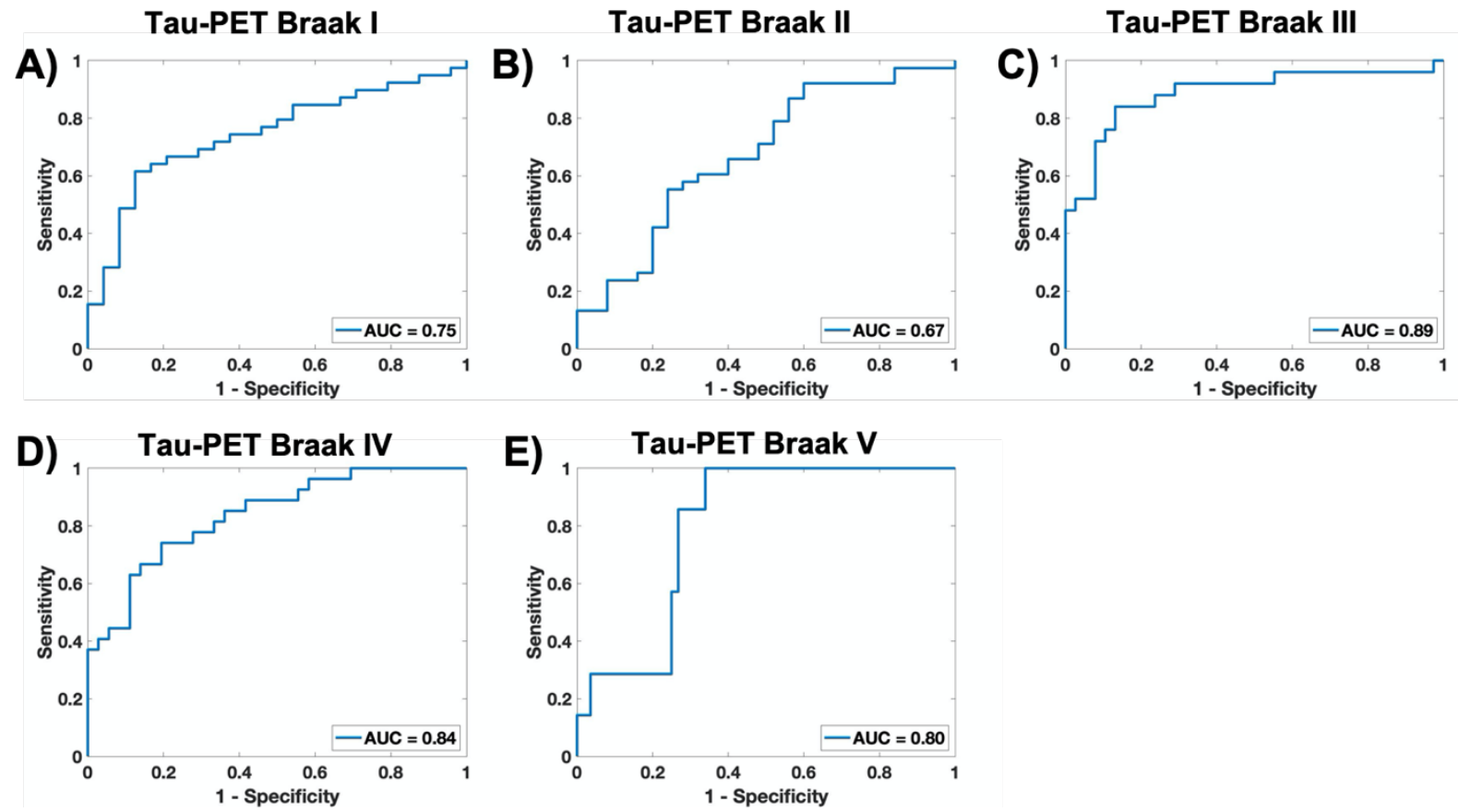

SUPPLEMENTAL FIGURE 5. Hippocampal efAP predicts tau positivity tau PET Braak regions. Receiver operating characteristics (ROC) curve performed using efAP as predictor variable and tau PET Braak region as the outcome variable. $(A)$ through $(E)$ represent the regions for Braak stages I through V, respectively. Area under the curve (AUC) shown for each of the five relationships. Since only one subject was tau-positive in region corresponding to Braak VI, ROC curve not shown. 